\title{
PREPARACIÓN DE NANOPARTÍCULAS DE PLATA EN AUSENCIA DE POLIMEROS ESTABILIZANTES
}

\author{
Ángela B. Sifontes, Luis Melo*, Carlos Maza, Juan J. Mendes y Marta Mediavilla \\ Facultad de Ingeniería, Universidad Central de Venezuela, P.O. Box 48.057, Caracas 1041-A, Venezuela \\ Joaquín L. Brito, Tamara Zoltan y Alberto Albornoz \\ Centro de Química, Instituto Venezolano de Investigaciones Científicas, P.O. Box 20.632, Caracas 1020-A, Venezuela
}

Recebido em 5/8/09; aceito em 24/2/10; publicado na web em 10/6/10

\begin{abstract}
PREPARATION OF SILVER NANOPARTICLES IN THE ABSENCE OF POLYMER STABILIZERS. Silver nanoparticles (AgNPs) were prepared by means of the polyol method in the absence of stabilizing polymers. To accomplish this objective, $\mathrm{AgNO}_{3}$ was added to ethylene glycol in the presence of $\mathrm{NaOH}\left(1 \mathrm{~mol} . \mathrm{L}^{-1}\right)$, the suspension formed was irradiated with a microwave source for 60 seconds at a power of 465 watts. It was found that under these conditions AgNPs of sizes between 4-18 nm are formed. Also the results indicate that part of the ethylene glycol is oxidized to carbonyl compounds that reduce the $\mathrm{Ag}^{+}$. These organic compounds are adsorbed on the surfaces of AgNPs, forming a protective film that prevents their aggregation.
\end{abstract}

Keywords: nanoparticles; polyol method; silver.

\section{INTRODUCCIÓN}

Recientemente la preparación de nanopartículas metálicas ha despertado gran interés en la comunidad científica, debido a las propiedades catalíticas, ópticas, electrónicas, magnéticas y bactericidas que presentan estos sistemas, lo que significa que son de gran utilidad tecnológica, siendo de especial atención los materiales que contienen nanopartículas de metales nobles, tales como: oro, ${ }^{1,2}$ plata, ${ }^{3,4}$ platino ${ }^{5-7}$ y paladio. ${ }^{8}$

Las nanopartículas metálicas se pueden definir como agregados aislados de tamaños entre 1 y $50 \mathrm{~nm}$, los cuales son un conjunto de átomos rodeados de una cápsula protectora o estabilizadora, que evita la aglomeración..$^{9,10}$

Existen varios métodos para la preparación de nanopartículas; en el caso de los nanoclusters o "nanoagregados" de los metales de transición se pueden citar: ${ }^{11}$ reducción química de una sal metálica; ${ }^{12,13}$ descomposición térmica, fotoquímica o sonoquímica; ${ }^{14,15}$ reducción del ligando ${ }^{11}$ y desplazamiento del ligando en compuestos organometálicos; deposición del metal en fase de vapor ${ }^{11} \mathrm{y}$ síntesis electroquímica. ${ }^{16}$

Uno de los métodos más comunes para la preparación de las nanopartículas, es la reducción química de sales de metales de transición en presencia de agentes estabilizadores. ${ }^{17,18} \mathrm{El}$ mecanismo de formación de nanopartículas se basa primordialmente, en la reducción de la sal metálica al átomo cerovalente y seguidamente, estos átomos actúan como centros de nucleación dando lugar a la formación de clusters cuyo crecimiento continuará a medida que se sigan agregando los átomos, formándose así, partículas de mayor tamaño y formas poliédricas más complejas. ${ }^{18}$ No obstante, es necesario estabilizar las partículas mediante la envoltura de moléculas o "agentes" estabilizadores (polímeros generalmente) que se adsorben en su superficie, inhibiendo de esta manera el proceso de aglomeración o sinterización. ${ }^{19-22}$

La principal ventaja del método de reducción química es su reproducibilidad y la posibilidad de obtener coloides monodispersos con una distribución estrecha en el tamaño de las partícula, ${ }^{12}$ razón por la cual, son frecuentemente preparadas nanopartículas metálicas siguiendo esta metodología, empleando como agentes reductores especies químicas tales como: ácidos orgánicos, alcoholes, polioles, aldehídos y azúcares. ${ }^{12}$

\footnotetext{
*e-mail: meloher@gmail.com
}

En los últimos años, el proceso de calentamiento asistido por microondas, se ha utilizado como una alternativa atractiva para la síntesis de materiales a escala nanométrica, dado que es un método rápido, uniforme y efectivo, que permite incrementar las cinéticas de reacción en uno o dos órdenes de magnitud. ${ }^{14,15}$ Nanopartículas coloidales de Pt, Ru, Ag y Pd estabilizados por polímeros, han sido preparadas por calentamiento con microondas, a partir de las sales precursoras del metal disueltas en soluciones de etilenglicol (EG). ${ }^{22-24}$

Por otra parte, el calentamiento por microondas de las muestras líquidas, permite la disminución de las fluctuaciones de temperatura en el medio de reacción, proporcionando así, un entorno más homogéneo para la nucleación y el crecimiento de las partículas metálicas. ${ }^{22-24}$

La formación de AgNPs por el método poliol en presencia de $\mathrm{NaOH}$, ha sido investigada ${ }^{25-27}$ y se ha concluido que bajo estas condiciones se puede aumentar la velocidad de reducción de los iones del metal y estabilizar el coloide resultante. En este sentido, se ha sugerido como medio de síntesis el EG, debido a su alta constante dieléctrica (41,4 a $298 \mathrm{~K}$ ) y pérdida dieléctrica, por lo que puede ser calentado rápidamente por la radiación de microondas, logrando acelerar la velocidad de reducción de los iones metálicos y favoreciendo la formación de los núcleos metálicos. El EG se descompone, generando especies tales como $\left(\mathrm{CH}_{3} \mathrm{CHO}\right)$, que pueden reducir los iones metálicos a átomos, ${ }^{22-25}$ los cuales se aglomeran y forman las partículas. Al emplear el EG se producen reacciones químicas en el medio de síntesis tales como las siguientes:

$$
\begin{aligned}
& \mathrm{CH}_{2} \mathrm{OH}-\mathrm{CH}_{2} \mathrm{OH} \stackrel{\mathrm{NaOH}}{\triangle}-\mathrm{CH}_{3} \mathrm{CHO}+\mathrm{H}_{2} \mathrm{O} \\
& 2 \mathrm{CH}_{3} \mathrm{CHO}+2 \mathrm{Ag}^{+} \longrightarrow 2 \mathrm{Ag}^{0}+2 \mathrm{H}^{+}+\mathrm{CH}_{3} \mathrm{COCOCH}_{3}
\end{aligned}
$$

Frecuentemente se menciona en la literatura ${ }^{19-25}$ el empleo de agentes estabilizadores para la preparación de nanopartículas metálicas, p. ej., polivinilpirrolidona (PVP), pero los mismos parecen inhibir su actividad catalítica y otras propiedades como consecuencia de su adsorción superficial. Por ello es importante explorar metodologías de preparación que eviten el uso de estos estabilizadores. En este sentido, en el presente trabajo se realiza un estudio sobre las condiciones de formación de AgNPs, empleando un proceso poliol, con la variante de utilizar $\mathrm{NaOH}$ y un calentamiento mediante una fuente 
de irradiación de microondas, lo que permite evitar el empleo de agentes estabilizadores de tipo poliméricos en el proceso de síntesis.

\section{EXPERIMENTAL}

Los materiales empleados en el presente estudio incluyen: nitrato de plata (Aldrich, 99,9\%), hidróxido de sodio (Merck 99,9\%) y etilenglicol (J.T. Baker). Se prepararon suspensiones ${ }^{27}$ añadiendo 0,03; 0,05 y 0,07 mmoles de $\mathrm{AgNO}_{3}$ sólido a $15 \mathrm{~mL}$ de EG, empleando agitación ultrasónica, seguidamente se adicionó gota a gota y con agitación continua, $0,5 \mathrm{~mL}$ de una solución de $\mathrm{NaOH} 1 \mathrm{~mol} \cdot \mathrm{L}^{-1}$. Posteriormente, la muestra fue colocada en un horno de microondas durante $60 \mathrm{~s}$, utilizando una potencia de 465 vatios.$^{28} \mathrm{El}$ horno de microondas utilizado en la síntesis fue un equipo Samsung, modelo MW 1050 de 2,45 GHz.

Las AgNPs obtenidas fueron separadas de la suspensión por centrifugación. El producto fue lavado varias veces con una solución etanol-agua y centrifugado nuevamente. Seguidamente, se prepararon suspensiones coloidales de AgNPs (en agua) y se evaluaron sus propiedades ópticas por espectroscopía ultravioleta-visible. Los espectros de UV-vis se realizaron en un espectrofotómetro Hitachi U2010.

Las AgNPs formadas se caracterizaron mediante espectroscopía fotoelectrónica de rayos X (XPS) empleando un equipo ESCALAB 220i-XL spectrometer, VG Scientific, lo que permitió determinar el estado de oxidación de las especies químicas presentes en la suspensión. En tal sentido, se efectuó una evaporación lenta del solvente, a $60^{\circ} \mathrm{C}$ y posteriormente se procedió a efectuar un análisis del residuo sólido.

La evaluación por microscopía electrónica de transmisión se efectuó en un instrumento Hitachi CM-10 operado a $120 \mathrm{kV}$. La muestra fue embebida en resina epoxi y secada $60^{\circ} \mathrm{C}$. Luego se cortó en ultamicrotomo para su observación en el microscopio electrónico de transmisión.

\section{DISCUSIÓN DE RESULTADOS}

\section{Estudio del efecto de la concentración de $\mathrm{AgNO}_{3}$}

Se realizó un estudio por espectroscopía UV-vis a las muestras preparadas mediante la metodología de síntesis presentada, empleando 0,$03 ; 0,05$ y 0,07 mmoles de $\mathrm{AgNO}_{3}$ y una potencia de irradiación en el microondas de 465 vatios, en las cuales se analizó el efecto de la concentración de la sal de plata en la mezcla de reacción, manteniendo constante la relación de reactivos $(\mathrm{EG} / \mathrm{NaOH})$ y el tiempo de irradiación de microondas. Las curvas de los espectros de absorción UV-vis son mostradas en la Figura 1. Esta técnica de caracterización permite correlacionar las bandas de absorción observadas debidas a la resonancia del plasmón superficial, con el tamaño y las formas de las AgNPs en estudio. La resonancia del plasmón superficial es originada por los electrones libres presentes en la superficie de las nanopartículas, los cuales interaccionan con la radiación UV. El efecto físico de la absorción de la luz por las AgNPs metálicas suspendidas en los líquidos, es la oscilación coherente de los electrones de la banda de conducción como respuesta a la interacción con el campo electromagnético, lo que es conocida como Resonancia de Plasmones de Superficie (RPS). ${ }^{29-31}$ Los espectros obtenidos presentan una banda característica a la RPS resultante de la oscilación colectiva de los electrones de conducción de la plata en las nanopartículas. ${ }^{32-35}$

En las gráficas de la Figura 1 se pueden observar bandas de resonancia de plasmón superficial de fuerte intensidad, en el rango 350$450 \mathrm{~nm}$. En el caso de las AgNPs, el pico de absorción del plasmón aparece normalmente a una longitud de onda alrededor de los 400 nm. ${ }^{29-33,37-40} \mathrm{El}$ ensanchamiento y la asimetría de los picos observados, puede ser atribuido a la formación de AgNPs de diferentes tamaños y formas $;{ }^{34}$ pero también pudiera estar indicándonos que esta ocurriendo la agregación de las partículas metálicas de este sistema, ya que se

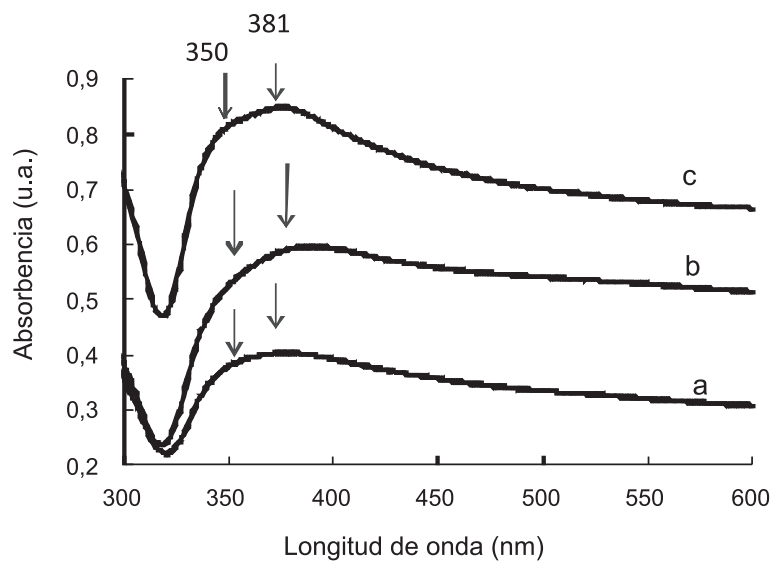

Figura 1. Espectro de absorción UV-visible para la muestra sintetizada con a) 0,03; b) 0,05; c) 0,07 mmoles de $\mathrm{AgNO}_{3}$; 0,0005 moles de $\mathrm{NaOH}$; 0,27 moles de EG y 465 vatios de potencia

observa absorción en el rango 450-600nm, dado que su posición exacta depende de varios factores, tales como el diámetro, la forma y la distribución de tamaños de las AgNPs formadas. ${ }^{29-33,36-40}$

Como puede observarse en la Figura 1, se aprecian distribuciones anchas con un máximo centrado hacia los $380 \mathrm{~nm}$ y un hombro alrededor de $\operatorname{los} 350 \mathrm{~nm}$, las cuales son atribuidas a la presencia de estructuras nanométricas de simetría no muy bien definida. ${ }^{34-38}$ No obstante, la resonancia del plasmón de superficie para AgNPs de morfología esférica, es frecuentemente observada en el rango de 380-430 nm. ${ }^{38}$

El hombro alrededor de $348 \mathrm{~nm}$ también pudiera ser atribuido a una banda de resonancia transversal, correspondiente a AgNPs con morfología de nanoplatos. ${ }^{39,40}$ Recientemente se ha mencionado ${ }^{39,40}$ que existen dos modos de resonancia de plasmón para las AgNPs con formas de nanoplatos: el modo transversal encontrado a menores longitudes de onda $(340 \mathrm{~nm})$ y el modo longitudinal ubicado alrededor de los $470-550 \mathrm{~nm}$, correspondiente a nanoplatos de mayores tamaños. ${ }^{39,40}$

\section{Efecto de la ausencia de $\mathrm{NaOH}$ en el medio de síntesis}

A los efectos de comparar los resultados de las distintas condiciones de síntesis de las AgNPs metálicas, se muestran en la Figura 2 los espectros UV-vis para las suspensiones preparadas con a) 0,03; b) 0,05 ; y c) 0,07 mmoles de $\mathrm{AgNO}_{3}$, en ausencia de $\mathrm{NaOH}$.

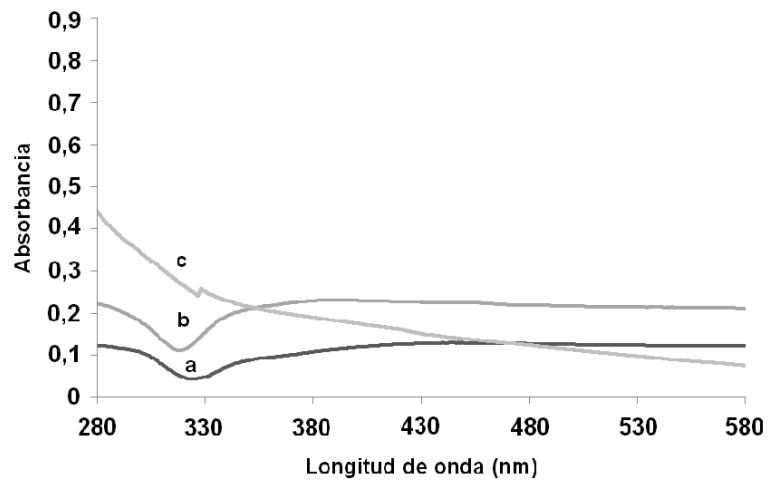

Figura 2. Espectro de absorción UV-visible para la muestra sintetizada con a) 0,03; b) 0,05; y c) 0,07 mmoles de $\mathrm{AgNO}_{3}$; 0,27 moles de EG, en ausencia de $\mathrm{NaOH}$ en el medio de síntesis

Observamos que la Figura 2 presenta formas muy diferentes respecto a las obtenidas de las suspensiones (EG/NaOH), apreciándose una fuerte disminución de intensidad en la señal de absorbancia de 
los plasmones, indicando un bajo rendimiento en la generación de AgNPs, lo que sin duda es atribuible a la ausencia del agente reductor ${ }^{41}$ como lo deberían ser el $\mathrm{NaOH}$ y el acetaldehído.

En consecuencia el método de síntesis presentado requiere suspender la sal de plata $\left(\mathrm{AgNO}_{3}\right)$ en un poliol ${ }^{27}(\mathrm{EG})$ y adicionarle una base $(\mathrm{NaOH})$, la suspensión formada es calentada mediante una fuente de irradiación de microondas durante $60 \mathrm{~s}$. La adición de una base fuerte permite formar una especie óxido intermediario de $\mathrm{Ag}(\mathrm{I})$ (térmicamente inestable). Especie intermediaría que es subsecuentemente reducida a plata metálica, mediante la acción del acetaldehído formado en el medio de síntesis (Ecuación 1 y 2) y el tratamiento térmico empleado. Sin embargo se entiende, que generalmente el óxido de plata es reducido térmicamente a temperaturas ${ }^{27}$ entre 200 y $500{ }^{\circ} \mathrm{C}$, lo que marca una gran diferencia con el procedimiento utilizado en este trabajo, donde por irradiación con microondas se alcanza una temperatura cercana a $90{ }^{\circ} \mathrm{C}$ y se logra el objetivo de producir las AgNPs.

\section{Estudio por microscopía electrónica de transmisión (TEM)}

La morfología de las AgNPs metálicas fue observada empleando la microscopía electrónica de transmisión. En la Figura 3 se muestran las imágenes de los productos obtenidos de la síntesis de AgNPs empleando 0,07 mmoles de $\mathrm{AgNO}_{3}$ después de haber sido irradiadas por microondas a una potencia de 465 vatios. En la micrografía presentada se aprecia claramente la morfología esferoidal de las partículas obtenidas, las cuales presentan diámetros en el orden de 4-18 nm y no se distinguen en ellas la presencia de nanoplatos, lo que esta en concordancia con la evaluación efectuada mediante UV-vis.

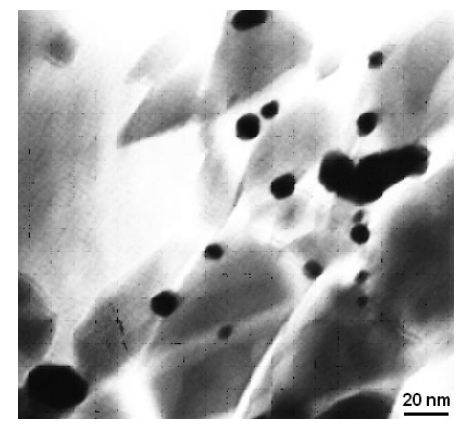

Figura 3. Microscopía electrónica de transmisión (TEM) de las AgNPs formadas en una suspensión preparada con 0,07 mmoles de $\mathrm{AgNO}_{3}$; 0,0005 moles de NaOH; 0,27 moles de EG y 465 vatios de potencia

\section{Estudio por espectroscopía fotoelectrónica de rayos X (XPS)}

Finalmente, se realizó la caracterización de las AgNPs presentes en la suspensión preparada con 0,07 mmoles de $\mathrm{AgNO}_{3}$ y una potencia de 465 vatios en el horno de microondas, mediante la técnica de espectroscopía fotoelectrónica de rayos X (XPS). En la Figura 4 se presenta el espectro XPS de alta resolución en la región espectral de Ag3d.

En la gráfica se evidencian señales a 374,2 y 368,7 eV las cuales son asignadas a los estados de $\mathrm{Ag} 3 \mathrm{~d}_{3 / 2}$ y $\mathrm{Ag} 3 \mathrm{~d}_{5 / 2}$, respectivamente, energías de enlace que confirman la presencia de plata en estado reducido $\left(\mathrm{Ag}^{0}\right),{ }^{42,43}$ resultados que están en plena coherencia con los obtenidos por UV-vis.

En la Figura 5, se observa el espectro XPS en la región correspondiente al C1s (284 a $290 \mathrm{eV}$ ), donde es posible identificar cuatro señales. ${ }^{44,45}$ Una primera a $284,5 \mathrm{eV}$, la cual puede ser atribuida al C1s de enlaces CHn/C-C, una segunda señal es identificada a $286,4 \mathrm{eV}$, que se asocia con el $\mathrm{C} 1$ s de enlaces $\underline{\mathrm{C}}-\mathrm{OH}$; la tercera señal aparece

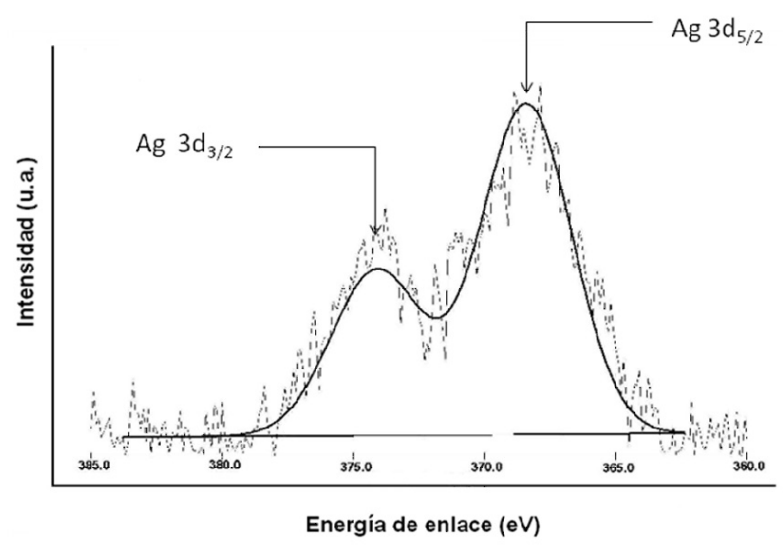

Figura 4. Espectro de XPS en la región correspondiente a la Ag3d para la suspensión de AgNPs preparadas con 0,07 mmoles de $\mathrm{AgNO}_{3}$; 0,0005 moles de NaOH; 0,27 moles de EG y 465 vatios de potencia

cercano a los 288,2 eV, que se atribuye al C1s de carbonos carbonílicos en los enlaces tipo $\mathrm{O}=\mathrm{C}-\mathrm{O}$ y finalmente aparece una cuarta señal a $290 \mathrm{eV}$, la cual es atribuida al C1s de los enlaces -COOR..$^{43,46}$

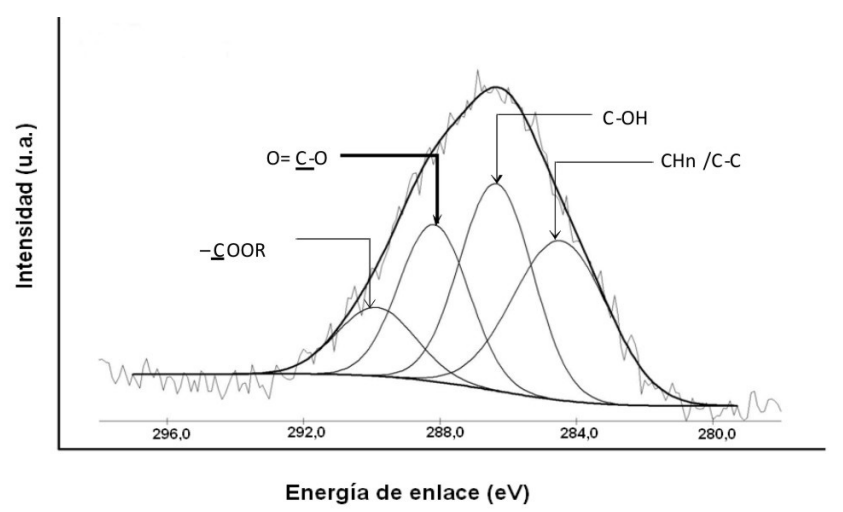

Figura 5. Espectro de XPS en la región correspondiente del C1s $(284$ a 290 eV) para la suspensión de AgNPs preparadas con 0,07 mmoles de $\mathrm{AgNO}$; 0,0005 moles de NaOH; 0,27 moles de EG y 465 vatios de potencia

Estos resultados parecen indicar de manera contundente la existencia de moléculas orgánicas adsorbidas sobre la superficie de las AgNPs metálicas, las cuales poseen las características que indica el análisis por XPS, y que pueden atribuirse a la presencia de etilenglicol, ácidos carboxílicos y esteres.

En la Figura 6, se muestra el espectro XPS en la región O1s, (528 a $543 \mathrm{eV}$ ) para las AgNPs obtenidas de la suspensión preparada con 0,07 mmoles de $\mathrm{AgNO}_{3}$ y una potencia de 465 vatios en el horno de microondas. Como puede observarse, se encuentra una señal a 531,4 eV, asociada al oxígeno doblemente enlazado a grupos $\mathrm{C}=\underline{\mathrm{O}},{ }^{44-46}$ igualmente se encuentra otra señal en $533,3 \mathrm{eV}$, atribuida a los oxígenos unidos a los grupos $\mathrm{O}=\mathrm{C}-\underline{\mathrm{O}}$ y C- $\underline{\mathrm{O}}^{4-46}$ Estos resultados se correlacionan perfectamente bien con los presentados en la Figura 5 y sugieren que parte del EG utilizado como solvente en este trabajo, es oxidado a compuestos carbonílicos

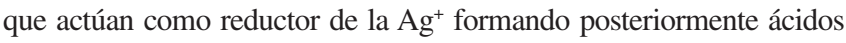
carboxílicos que pudieran dar origen a la formación de esteres durante el proceso de reducción, tal y como lo han señalado Bonet y colaboradores ${ }^{47}$ y se encuentran adsorbidos sobre la superficie de las AgNPs sintetizadas, actuando como agentes protectores, impidiendo la formación de agregados metálicos de mayores dimensiones. Esta situación es similar pero no exactamente igual a la estabilización de las nanopartículas con polímeros tales como la polivinilpirrolidona (PVP) añadidos ex profeso al medio 
de síntesis. Aunque este último caso produce, en general, la pasivación de los partículas metálicas para su empleo, p. ej., como catalizadores, el EG y sus derivados orgánicos parecen ser menos negativos para el uso de las nanopartìculas en catálisis. Esta hipótesis, por supuesto, debe ser investigada en estudios posteriores.

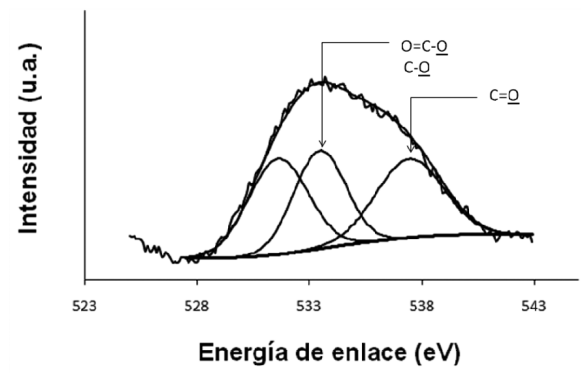

Figura 6. Espectro de XPS en la región del O1s, (528 a 543 eV) para la suspensión de AgNPs preparadas con 0,07 mmoles de $\mathrm{AgNO}_{3}$; 0,0005 moles de NaOH; 0,27 moles de EG y 465 vatios de potencia

\section{CONCLUSIONES}

Se ha desarrollado un método rápido para la obtención de nanopartículas de plata mediante el empleo de un proceso poliol, en ausencia de polímeros o surfactantes, utilizando un calentamiento con microondas durante $60 \mathrm{~s}$.

Luego de la aplicación del etilenglicol, $\mathrm{NaOH}$ y calentamiento con microondas, se observó la aparición de una banda de absorción en el espectro UV-vis, cuyos máximos indican la formación de nanopartículas de plata de diferente morfología.

Los estudios por XPS confirman la presencia de plata en estado reducido $\left(\mathrm{Ag}^{0}\right)$ y la existencia de compuestos orgánicos tales como: alcoholes (EG), ácidos y esteres adsorbidos sobre la superficie de las AgNPs metálicas, las cuales pudieran actuar como películas protectoras que impiden la formación de partículas de mayores dimensiones.

El método propuesto permite la formación de AgNPs metálica a temperaturas menores a los $100{ }^{\circ} \mathrm{C}$ y morfología esferoidal, con diámetros promedio entre 4 y $18 \mathrm{~nm}$.

\section{AGRADECIMIENTOS}

Los autores agradecen al Proyecto CDCH-UCV (PG-O8-006075 2005) y al Instituto Venezolano de Investigaciones Científicas (IVIC) por el soporte financiero y técnico prestado en la realización de ésta investigación.

\section{REFERENCIAS}

1. Yu, Y.; Chang, S. S.; Lee, C. L.; Wang, C. R. C.; J. Phys.Chem. B 1997, $101,6661$.

2. Mohamed, M. B.; Ismail, K. Z.; Link, S.; El-Sayed, M. A.; J. Phys. Chem. B 1998, 102, 9370.

3. Rodrigues-Sanchez, L.; Blanco, M. C.; Lopez-Quintela, M. A.; J. Phys. Chem. B 2000, 104, 9683.

4. Yin, S.; Ma, H.; Wang, S.; Chen, S.; J. Phys. Chem. B 2003, 107, 8898.

5. Ma, H.; Yin, B.; Wang, S.; Jiao, Y.; Pan, W.; Chen, S.; Huang, S.; Meng, F.; ChemPhysChem 2004, 5, 68.

6. Ascarelli, P.; Contini, V.; Giorgi, R. J. ; Appl. Phys. 2002, 91, 4556

7. Mizukoshi, Y.; Takag, E.; Okuno, H.; Oshima, R.; Maeda, Y.; Nagata, Y.; Ultrasonics Sonochemistry 2001, 8, 1.

8. Pergolese, B. M.; Muniz-Miranda, B.; Bigotto, A.; Chem. Phys. Lett. 2007, 438, 290.

9. Lewis, L. N.; Chem. Rev. 1993, 93, 2693.
10. Richards, R. M.; Eur. J. Inorg. Chem. 2001, 10, 2455.

11. Bradey, J .S.; Schmid, G.; Clusters and Colloid, From Theory to Applications, VCH: Weinheim, 1994, p. 523.

12. Bonet, F.; Grugeon, S.; Herrera-Urbina, R.; Tekeia-Elhsissen, K.; Tarascon, J. M.; Solid State Sci. 2002, 4, 665.

13. Sun, J.; Jing, Y.; Jia, Y.; Belin, M. T. C.; Mat. Lett. 2005, 59, 3933.

14. Jiang, L.; Wang, A.; Zhao,Y.; Zhang, J.; Zhu, J.; J. Inorg. Chem. Commun. 2004, 7, 506.

15. Lu, H.; Liu, X. L.; Wang, X. F.; Qian, X. F.; Yin, J.; Zhu, Z. K.; Mat. Lett. 2003, 81, 104.

16. Faraday, M.; Philos. Trans R. Soc. London 1857, 14, 145.

17. Wiley, B.; Sun, Y.; Mayers, B.; Xia. Y.; Chem.- A Eur. J. 2005, 11, 454.

18. Turkevich, J.; Kim, G.; Science 1970, 169, 873.

19. Mu, X.; Evans, D.; Kou, Y.; Catal. Lett. 2004, 97,151.

20. Liu, Y.; Chen, S.; Zhong, L.; Wu, G.; Rad. Phys. Chem. 2009, 78, 251.

21. Wook Kan, S.; Hong, J.; Park, J.; Mun, S. H.; Kim, J. H.; Cho, J.; Char, K.; Kang, Y. S.; J. Memb. Sci. 2008, 321, 90.

22. Patel, K.; Kapoor, S.; Dave, D.; Murkherjee, T.; J. Chem. Sci. 2005, 117, 53.

23. Zhu, H.; Zhang, C.; Yin, Y.; J. Crys. Growth. 2004, 270, 722.

24. Chen, W.; Lee, J. Y.; Liu, Z.; Mater. Lett. 2004, 58, 3166.

25. Chou, K.; Lai, Y.; Mater. Chem. Phys. 2004, 83, 82.

26. He, B.; Tan, J.; Liew, K.; Liu, H.; J. Mol. Catal. A 2004, 221,121.

27. Goia, D. V.; Fritzsche, S.; Kempf, B.; Braumann, P.; Vandevelde, T.; US pat. 112926, 2007.

28. da Silva, F.; Ferreira, F. V.; de Souza, M.; Quim. Nova 2006, 29, 376.

29. Kelly, K. L.; Coronado, E.; Zhao, L. L.; Schatz, G. C.; J. Phys. Chem. B 2003, 107, 668 .

30. Sosa, I. O.; Noguez, C.; Barrera, R. G.; J. Phys. Chem. B 2003, 107, 6269.

31. Mizukoshi, Y.; Fujimoto, T.; Nagata, Y.; Oshima, R.; Maeda, Y.; J. Phys. Chem. B 2000, 104, 6028

32. Mulvaney, P.; Langmuir 1996, 12, 788.

33. van der Zande, B. M. I.; Böhemer, M. R.; Fokkink, L. G. J.; Schönenberger, C.; Langmuir 1999, 16, 451.

34. Kottmann, J. P.; Martin, O. J. F.; Smith, D. R.; Schultz, S.; Phys. Rev. B 2001, 64, 235402.

35. Cai-Xia, K.; Zhu, J.; Zhu, X.; J. Phys. D: Appl. Phys. 2008, 41, 155304.

36. Petit, C.; Lixon, P.; Pileni. M. P.; J. Phys. Chem. 1993, 97, 12974.

37. Mock, J. J.; Barbic, M.; Smith, D. R.; Schultz, D. A.; Schultz. S.; J. Chem. Phys. 2002, 116, 6755.

38. Mohanty, J.; Palit, D.; Shastri, L. V.; Sapre, A. V.; Proc. Indian Acad. Sci. 2000, 112, 63.

39. Cheng, J.; Wang, J.; Zhang, X.; Jin, Y.; Mater. Chem. Phys. 2008, 108, 421.

40. Tian, X.; Wang, W.; Cao, G.; Mat. Lett. 2007, 61, 130.

41. Zhou1, M.; Wang, B.; Rozynek, Z.; Xie, Z.; Nanotechnology 2009, 20 , 505606.

42. Sharma, J.; Chaki, N. K.; Mandale, A. B.; Pasricha, R.; Vijayamohanan, K.; J. Colloid Interface Sci. 2004, 172, 145.

43. Moulder, J. F.; Stickle, W. F.; Sobol, P. E.; Bomben, P. E.; Handbook of $X$-Ray Photoelectron Spectroscopy, $2^{\text {nd }}$ ed., Perkin-Elmer: Eden Prairie, 1992.

44. Heister, K.; Johansson, L. S. O.; Grunze, M.; Zharnikov, M.; Surf. Sci. 2003, 529, 36.

45. Pijpers, A. P.; Meier, R. J.; Chem. Soc. Rev. 1999, 28, 233.

46. Rjeba, B. M.; Labzourb, A. M.; Rjebb, A.; Sayouric, S.; Chafik, M.; Idrissid, E.; Masseye, S.; Adnote, A.; Roye, D.; J. Phys: Condens. Matter 2004, 5,168.

47. Bonet, F.; Tekaia-Elhsissen, K.; Vijaya Sarathy, K.; Bull. Mater. Sci. 2000, 23, 165 . 